\title{
Partial Response in cGVHD
}

National Cancer Institute

\section{Source}

National Cancer Institute. Partial Response in CGVHD. NCI Thesaurus. Code C103140.

Improvement in one or more organ or tissue without prog ression in other affected

organs or tissue in chronic Graft versus Host Disease (cGVHD). 